\title{
Highlights from the Twentieth Annual Transcatheter Cardiovascular Therapeutics Meeting
}

Mathew Williams, MD

The 20th Annual Transcatheter Cardiovascular Therapeutics (TCT) Meeting, TCT 2008, was held in Washington, DC, October 12 through 17, 2008. TCT is the largest meeting in the world devoted to transcatheter therapies and is predominantly an interventional cardiology meeting. For the second year, a program was offered for cardiac surgeons to help bring the specialty into this rapidly progressing and exciting field. Although there were many significant trials, mostly pertaining to the treatment of coronary artery disease (as described here), the meeting focused extensively on demonstrating procedures and highlighting new technologies.

\section{TRANSCATHETER VALVE THERAPY}

One of the better-attended sessions was the valvular heart disease summit. This included an excellent review of current surgical procedures but focused mainly on emerging transcatheter techniques. The summit provided updated experiences with current devices, as well as first human trials and preliminary work for many devices on the horizon. Although the data are largely encouraging, most work is preliminary and not really appropriate for discussion until more data become available. Two systems are currently approved for use in Europe and have been applied with variable but generally encouraging success.

\section{CoreValve}

The experience from Siegburg, Germany, with the $18 \mathrm{~F}$ self-expanding CoreValve system (CoreValve Inc, Irvine, Calif) was presented. ${ }^{1}$ Implant success was $97.1 \%$, with no procedural mortality. The 30-day mortality was $10.8 \%$, with a stroke rate of $2.9 \%$. The 1-year survival was $84.3 \%$. No patient had residual aortic stenosis, and all had improvement in symptoms. Relative to baseline, $34.6 \%$ of patients had a reduction of aortic insufficiency, $34.6 \%$ had no change, and $30.9 \%$ had worsening of aortic insufficiency.

\section{SAPIEN Valve}

An experience with the SAPIEN transcatheter aortic valve (Edwards Lifesciences LLC, Irvine, Calif) was presented from Vancouver, BC, Canada, the site with the largest worldwide experience. ${ }^{2}$ The data were generally impressive.

\footnotetext{
From the Department of Surgery and Medicine, Columbia University, College of Physicians and Surgeons, New York, NY.

Received for publication Nov 19, 2008; accepted for publication Nov 19, 2008.

Address for reprints: Mathew Williams, MD, College of Physicians and Surgeons of Columbia University, Department of Surgery and Medicine, 177 Ft. Washington Ave, MHB 7-435, New York, NY10032 (E-mail: mw365@columbia.edu). J Thorac Cardiovasc Surg 2009;137:509-11 0022-5223/\$36.00

Copyright (c) 2009 by The American Association for Thoracic Surgery doi:10.1016/j.jtcvs.2008.11.013
}

In particular, the center broke down their 114 transfemoral cases into tertiles. The first tertile had an implant success of $81.6 \%$, which improved in both the second and third tertiles to $97.4 \%$. The 30 -day mortality in this same group went from $13.2 \%$ in the first tertile to $10.5 \%$ in the second and $0 \%$ in the third.

\section{TRAVERSE}

TRAVERSE $^{3}$ is the European feasibility study of the transapical approach with the Edwards transcatheter aortic valve. There were 168 patients in a 3.5-year period. The implant success was $92.8 \%$. Cardiopulmonary bypass was used in $23 \%$ of cases, and $7.1 \%$ of patients required conversion to a sternotomy. The 30 -day survival was $85 \%$, and the 6-month survival was $70 \%$. This experience compared favorably with the US REVIVAL II transapical experience (6-month survival 64\%) and particularly the PARTNER EU Transapical experience (6-month survival 56\%).

\section{AMADEUS}

AMADEUS $^{4}$ (cArillon Mitral Annuloplasty Device European Union Study) is a feasibility trial evaluating the CARILLON device (Cardiac Dimensions Inc, Kirkland, Wash). ${ }^{4}$ CARILLON is a mitral annuloplasty device implanted percutaneously into the coronary sinus. The device can be retrieved during the procedure if there is no acute success. Among the 43 patients in whom placement was attempted, 30 received permanent devices because of acute reduction in mitral regurgitation (mitral regurgitation from grade 3 to $2, P<.001)$. The coronary artery was crossed in $84 \%$ of patients but only caused compromise necessitating device removal in 6 patients. In the last 10 patients, the procedure time averaged 10 minutes.

\section{MANAGEMENT OF CORONARY ARTERY DISEASE}

Most of the major trials presented at TCT pertained to percutaneous coronary intervention (PCI) for coronary artery disease. Some of the more relevant are described here.

\section{SYNTAX}

The SYNTAX trial ${ }^{5,6}$ (SYNergy between PCI with TAXUS and Cardiac Surgery) was a randomized trial comparing PCI with TAXUS drug-eluting stents (Boston Scientific Corporation, Natick, Mass) against coronary artery bypass grafting $(\mathrm{CABG})$ in patients with complex coronary artery disease. The results had previously been presented at the European Society of Cardiology. At the TCT meeting, two more in-depth subanalyses were provided to further 
delineate the left main $(\mathrm{CABG} n=345, \mathrm{PCI} n=528)$ and triple-vessel $(\mathrm{CABG} \mathrm{n}=549$, PCI $\mathrm{n}=546)$ groups. It is important to note that these data only provide outcomes at 1 year.

In the patients with unprotected left main disease the incidences of major adverse cardiac and cerebrovascular events (MACCEs) at 1 year was similar for CABG and PCI (13.6\% vs $15.8 \%, P=.44)$. Patients undergoing $C A B G$ had a lower rate of repeat revascularization $(6.7 \%$ vs $12.0 \%, P=.02)$, but at the expense of a significantly higher stroke rate $(2.7 \%$, vs $0.3 \%, P=.009)$. If these patients were broken down by the complexity of their lesions (SYNTAX score), there was no difference in MACCE rate between CABG and PCI in the cases of low and intermediate complexity. In the high-complexity cases, the MACCE rate was significantly higher in the PCI group $(12.9 \%$ vs $25.3 \%, P=.008)$.

In patients with triple-vessel disease, the MACCE, myocardial infarction (MI), and repeat revascularization rates were all higher in the PCI group (Table 1). The exception to this was the group with a low SYNTAX score, in which MACCE rates were similar for PCI and CABG.

\section{FAME}

The FAME trial ${ }^{7}$ (Fractional Flow Reserve versus Angiography for Guiding PCI in Patients with Multivessel Coronary Artery Disease) randomly assigned patients with multivessel disease to undergo either angiographically guided PCI or fractional flow reserve (FFR)-guided PCI. FFR is a hemodynamic rather than visual assessment of a lesion. It essentially compares the pressure on either side of a lesion during maximal hyperemia (achieved with adenosine) by advancing a special wire with a small pressure transducer across a lesion. Any lesion with a postlesion/prelesion ratio lower than 0.80 is considered significant. A total of 1000 patients were randomly assigned (angiographically guided $\mathrm{n}=496$, FFR guided $\mathrm{n}=509$ ). Patients in the FFR group had a reduced number of stents used, less contrast agent used, and lower overall cost. More importantly, they also had lower rates of major adverse cardiac events (MACEs, $13.2 \%$ vs $18 . \%, P=.02$ ) and of death or MI $(7.3 \%$ vs $11.1 \%, P=.04)$.

\section{HORIZONS AMI}

HORIZONS $\mathrm{AMI}^{8}$ (Harmonizing outcomes with Revascularization and Stents in Acute Myocardial Infarction) was a study with two primary purposes. The first was to demonstrate the optimal anticoagulation regimen for patients having PCI for AMI. The second was to compare the use of bare metal against that of drug-eluting stents in the same population. The anticoagulation arm had been published and presented previously (TCT 2007) and demonstrated the use of bivalirudin alone as superior to the use of heparin with a glycoprotein IIb/IIIa inhibitor. ${ }^{9}$ A total of 3000 patients had been enrolled in the stent arm of the trial, which used either a TAXUS drug-eluting stent or an
TABLE 1. Triple-vessel disease: Outcomes at 1 year

\begin{tabular}{lccc}
\hline \multicolumn{1}{c}{ End point } & CABG $(\mathbf{n}=\mathbf{5 4 9})$ & PCI $(\mathbf{n = 5 4 6})$ & $\boldsymbol{P}$ value \\
\hline MACCE & $11.2 \%$ & $19.1 \%$ & $<.001$ \\
Myocardial infarction & $2.6 \%$ & $5.2 \%$ & .04 \\
Repeat revascularization & $5.4 \%$ & $14.7 \%$ & $<.001$ \\
\hline
\end{tabular}

$C A B G$, Coronary artery bypass grafting; $P C I$, percutaneous coronary intervention; $M A C C E$, major adverse cardiac and cerebrovascular event.

Express bare metal stent (same platform but without drug and polymer, also from Boston Scientific), randomly allocated in a 3:1 fashion. There was no difference in overall MACE rate, which included stent thrombosis at 1 year, between drug-eluting and bare metal stent groups $(8.1 \%$ vs $8.0 \%, P=.92)$. Drug-eluting stents were associated with a significantly lower incidence of revascularization in the target lesion $(4.5 \%$ vs $7.5 \%, P=.002)$.

\section{BBC ONE}

BBC ONE ${ }^{10}$ (British Bifurcation Coronary Study: Old, New, and Evolving Stategies) was a randomized trial to evaluate ideal stent management for bifurcation lesions. A total of 500 patients were randomly allocated to two arms. Patients in the first group were treated with a simple strategy in which the main vessel lesion was stented and the side branch underwent angioplasty and provisional stenting. Those in the second group were treated with a complex strategy involving coverage of the entire lesion with stents in both branches through more complex techniques. The patients with the simple strategy had a lower periprocedural MACE rate $(2.0 \%$ vs $7.6 \%, P=.003)$ and also benefited from shorter procedures with less resource use. At 9 months of follow-up, the composite end point of death, MI, or target vessel failure was significantly lower with the simple strategy $(8.0 \%$ vs $15.2 \%, P=.009)$. The individual end points were also lower in the simple strategy, but only the MI end point achieved significance.

\section{MAIN-COMPARE}

MAIN-COMPARE ${ }^{11}$ (Revascularization for Unprotected Left Main Coronary Artery Stenosis: Comparison of Percutaneous Coronary Angioplasty vs Surgical Revascularization) is a registry study from Korea that compares CABG against PCI for left main coronary artery disease. An update including 3-year follow-up was provided from the registry. With propensity matching, there were no differences in survival, Q-wave MI, or stroke between CABG and PCI groups. Patients undergoing $\mathrm{CABG}$, however, had a significantly lower rate of revascularization at 3 years $(2.6 \%$ vs $9.3 \%, P<.001)$.

\section{COOL-RCN}

COOL-RCN ${ }^{12}$ (Cooling to Prevent Radio Contrast Nephropathy in Patients Undergoing Diagnostic or Interventional Catheterization) was a trial that used systemic hypothermia 
$\left(33^{\circ}-34^{\circ} \mathrm{C}\right)$ in hopes of preventing radiocontrast-induced nephropathy in patients at high risk for this complication. The trial therapy was randomly allocated against a normothermia $\mathrm{arm}$. The primary end point was radiocontrast-induced nephropathy at 96 hours. There were 70 patients in the normothermia arm and 58 patients in the hypothermia arm. The serum creatinine levels did not differ at baseline. There was no beneficial effect of hypothermia on serum creatinine at either 24 hours $(1.55 \mathrm{mg} / \mathrm{dL}$ vs $1.91 \mathrm{mg} / \mathrm{dL}, P=.30)$ or 48 hours $(1.94 \mathrm{mg} / \mathrm{dL}$ vs $2.21 \mathrm{mg} / \mathrm{dL}, P=.09)$. Contrast-induced nephropathy occurred in $18.6 \%$ of the control group and $22.4 \%$ of the hypothermia group $(P=.59)$. The study was limited by the inability to enroll the expected 400 patients and the fact that the study was based on an expected rate of nephropathy in the control group of $35 \%$.

\section{DECREASE}

DECREASE $^{13}$ is a registry study from South Korea that compares double antiplatelet therapy (aspirin and clopidogrel) against triple antiplatelet therapy (aspirin, clopidogrel and cilostazol) in patients receiving drug-eluting stents. The study was conducted on a single-center registry of 1443 patients receiving triple antiplatelet and 1656 patients receiving double antiplatelet therapy. Triple antiplatelet therapy was found to reduce significantly the 12-month risk of stent thrombosis (hazard ratio $0.108,95 \%$ confidence interval $0.013-0.918, P=.415$ ). Additionally, in an analysis of propensity-matched pairs, the thrombosis incidence in the triple therapy group was lower $(0.1 \%$ vs $0.8 \%, P=.0192)$. There was no difference in bleeding rate between the groups.

\section{PREPARE}

The PREPARE trial ${ }^{14}$ (Prospective Randomized Trial of Proximal Microcirculatory Protection in Patients with Acute Myocardial Infarction Undergoing Primary PCI) was a randomized trial in patients with ST-segment elevation MI that compared traditional therapy against proximal embolic protection with the Proxis device (St Jude Medical Inc, Minneapolis, Minn). The Proxis device is essentially a large-lumen catheter with a low-pressure balloon at the end. The catheter is placed in the infarct-related coronary artery, and the balloon is then inflated, which occludes proximal flow. In theory, this allows the lesion to be crossed and any thrombotic material to be aspirated, rather than breaking up the thrombus and allowing downstream embolization. There were 141 patients in the device arm and 143 in the control arm. The patients who received proximal embolic protection had immediate ST-segment resolution $66 \%$ of the time, versus $50 \%$ in the control group $(P=.009)$.

\section{CONCLUSION}

The TCT 2008 meeting had many significant studies, most of which are especially relevant to the practicing inter- ventional cardiologist. There were, however, many components directly or indirectly relevant to the cardiac surgeon. In the realm of coronary artery disease, the data are still relatively short term. In general, the role of cardiac surgery in complex multivessel disease is perhaps more solidified. Increasing amounts of data, however, suggest that PCI will play an increasing role in the treatment of left main coronary artery disease. The treatment of valvular heart disease with transcatheter techniques is evolving rapidly. Although probably many devices will fail, no doubt some will be successful. These devices will have a direct impact on our specialty and on the way that patients with valvular heart disease are treated. It is to be hoped that programs such as that offered at TCT will allow those in our specialty to become leaders with these new technologies, rather than victims of their success.

\section{References}

1. Gerkens U. Results with the CoreValve Transcatheter Heart Valve. Largest single center experience. Transcatheter Cardiovascular Therapeutics (TCT); 2008 Oct 12-17; Washington, DC.

2. Webb J. Results with the SAPIEN Transcatheter Heart Valve. Largest single center experience. Transcatheter Cardiovascular Therapeutics (TCT); 2008 Oct 12 17; Washington, DC

3. Walther $\mathrm{T}$ Update from the TRAVERSE European feasibility study. Transcatheter Cardiovascular Therapeutics (TCT); 2008 Oct 12-17; Washington, DC.

4. Siminiak T. AMADEUS: Feasibility study of the Carillon coronary sinus annuloplasty system for treatment of mitral regurgitation. Transcatheter Cardiovascular Therapeutics (TCT); 2008 Oct 12-17; Washington, DC.

5. Serruys PW. Revascularization in patients with unprotected left main coronary artery disease. New data from SYNTAX. Transcatheter Cardiovascular Therapeutics (TCT); 2008 Oct 12-17; Washington, DC.

6. Mohr FW. Revascularization in patients with triple-vessel coronary artery disease: new data from SYNTAX. Transcatheter Cardiovascular Therapeutics (TCT); 2008 Oct 12-17; Washington, DC.

7. Pijls NH. Fractional flow reserve versus angiography for guiding PCI in patients with multivessel coronary artery disease. Transcatheter Cardiovascular Therapeutics (TCT); 2008 Oct 12-17; Washington, DC.

8. Stone GW. Horizons AMI: A prospective randomized trial of paclitaxel-eluting stents vs. bare-metal stents in patients with acute ST-segment elevation myocardial infarction. Transcatheter Cardiovascular Therapeutics (TCT); 2008 Oct 1217; Washington, DC.

9. Stone GW, Witzenbichler B, Guagliumi G, Peruga JZ, Brodie BR, Dudek D, et al. Bivalirudin during primary $\mathrm{PCI}$ in acute myocardial infarction. $N$ Engl $\mathrm{J} \mathrm{Med}$. 2008;358:2218-30.

10. Hildick-Smith D. British Bifurcation Coronary Study: old, new and evolving strategies. Transcatheter Cardiovascular Therapeutics (TCT); 2008 Oct 12-17; Washington, DC.

11. Seung KB. MAIN-COMPARE Registry: stenting versus CABG in unprotected left main Disease. Transcatheter Cardiovascular Therapeutics (TCT); 2008 Oct 12-17; Washington, DC.

12. Stone GW. Cool RCN: a prospective randomized comparison systemic hypothermia versus normothermia for prevention of contrast induced nephropathy. Transcatheter Cardiovascular Therapeutics (TCT); 2008 Oct 12-17; Washington, DC.

13. Park SW. One year results comparing dual versus triple antiplatelet therapy in patient receiving drug-eluting stents. Report form the DECREASE registry Transcatheter Cardiovascular Therapeutics (TCT); 2008 Oct 12-17; Washington, DC.

14. Koch KT. PREPARE: a prospective randomized trial of proximal microcirculatory protection in patients with acute myocardial infarction undergoing primary percutaneous coronary intervention. Transcatheter Cardiovascular Therapeutics (TCT); 2008 Oct 12-17; Washington, DC. 